\title{
ETAT DES LIEUX ET IMPACTS SOCIO-ENVIRONNEMENTAUX DE LA GESTION DES DECHETS HOSPITALIERS SOLIDES DANS LA VILLE DE YAOUNDE, CAPITALE DU CAMEROUN
}

\author{
DJIYO Denis ${ }^{1,2}$, MWEBI EKENGOUE Clautaire ${ }^{2}$, MANGA Blaise Laurent ${ }^{3}$, TEOUGAM Nicolas $^{1}$, \\ HARRAG Abdelmalek ${ }^{4}$, AGOKENG DONGMO Gisele Mélanie ${ }^{5}$
}

\begin{abstract}
${ }^{1}$ Laboratoire du Génie de l'environnement, Ecole Nationale Supérieure Polytechnique de Maroua (ENSPM), Extrême-Nord Cameroun. B.P. 46 Maroua.

${ }^{2}$ Association Scientifique Africaine pour I'Innovation et l'Entrepreneuriat (ASAIE), Dschang, Ouest-Cameroun.

${ }^{3}$ Ministère de la santé publique (MINSANTE), Région du Centre Cameroun. B.P. 5680 Yaoundé.

${ }^{4}$ Département de Biologie et d’Ecologie Végétale, Faculté des Sciences de la Nature et de la vie, Université de Ferhat Abbas Sétif1, Sétif, Algérie.

${ }^{5}$ Unité de Recherche de Biochimie des Plantes Médicinales, des sciences Alimentaires et de Nutrition (URBPMAN), Département de Biochimie, Faculté des Sciences, Université de Dschang, Ouest-Cameroun.
\end{abstract}

Auteure correspondante : clautaire.mwebiekengoue@yahoo.fr

\section{RESUME}

Cet article présente l'état des lieux de la gestion des déchets hospitaliers (DHs) solides dans la capitale du Cameroun ainsi qu'une appréciation des impacts socioenvironnementaux associés. Les travaux se sont déroulés suivant 4 axes majeurs : (i) aperçu global de la gestion des $\mathrm{DH}$ s solides dans cinq formations sanitaires (FOSAs) de la ville de Yaoundé, (ii) quantité de DHs solides produite dans chaque FOSA, (iii) mode de gestion employé dans les différentes FOSAs et (iv) impacts sociaux et environnementaux correspondants. L'observation de terrain et le dépouillement des données de l'enquête menée auprès du personnel médical et des usagers ont montré que l'ensemble des DHs solides produits dans les FOSAs sélectionnées regroupe les déchets ménagers (76,79\%), pathologiques $(12,43 \%)$, piquants et tranchants $(9,58 \%)$, anatomiques $(0,60 \%)$ et spéciaux $(0,30 \%)$. Le système de gestion de ces déchets est encore à l'état embryonnaire du fait de l'absence d'un véritable plan de gestion des déchets, du manque d'information et de formation du personnel médical, du manque de personnels qualifiés en gestion des déchets mais aussi du manque de moyens financiers. En conséquence, on assiste à une mauvaise gestion des DHs solides, à chaque étape du processus de gestion des déchets, entrainant l'exposition des populations à de nombreux impacts sanitaires possibles. La pollution de l'environnement est également sensible du fait du dépôt des DHs sur le sol, leur transport par les eaux de ruissellement, mais aussi des émanations gazeuses résultant de leur décomposition.

MOTS-CLES : Yaoundé, centres de santé, déchets hospitaliers solides, impacts socio-environnementaux, gestion des déchets hospitaliers solides.

\begin{abstract}
This paper presents an overview on biomedical wastes (BWs) management in the capital city of Cameroon and related socio-environmental impacts. Four elements constitute major axes in this study: (i) the general view of BWs management in five health centers (HCs) in Yaoundé city, (ii) the among of BWs produced in each $\mathrm{HC}$, (iii) the management method used in various $\mathrm{HCs}$ and (iv) resulted social and environmental impacts. Field observations and data analysis collected from the medical staff and users have shown that BWs produced in those HCs of our choice regroup household waste (76.79\%), pathological waste (12.43\%), prickly and cutting waste $(9.58 \%)$, anatomical waste $(0.60 \%)$ and special waste $(0.30 \%)$. The management system of these wastes still in his embryonic state due to lack of real waste management plan, lack of information and training of medical staff, lack of qualified staff in waste management and also lack of financial issues. As consequences, one attends poor BWs management at each step of waste management, with consequence the exposure of the populations to numerous possible illnesses. The environmental pollution is also sensitive because of BWs deposit on soil, their transportation by water streaming and also of poisonous gas dismissal in air during their decomposition.
\end{abstract}

KEYWORDS: Yaoundé, health centers, biomedical wastes, social and environmental impacts, biomedical waste management. 


\section{INTRODUCTION}

La production de déchets et les systèmes de gestion mis en place sont caractéristiques du mode de vie des habitants de la société considérée (Béguin, 2013). De nombreuses techniques sont utilisées dans le monde pour traiter les déchets, telles que l'enfouissement, le compostage, l'incinération, l'utilisation dans l'alimentation animale, etc. (De Silguy, 2009). Les déchets hospitaliers ( $\mathrm{DH}$ ), nécessitent une gestion appropriée du fait de leur dangerosité. Ils ont fait l'objet de nombreux travaux de recherche tels que ceux de Béguin (2013), Datta et al. (2018), Nema et Ganesprasad (2002), Chartier et al. (2014), Li et Jenq (1993) et Balekouzou et al. (2016).

$\mathrm{Au}$ Cameroun, les dispositions législatives et règlementaires existent, mais leur mise en application est insuffisante. Dans certaines régions du Cameroun, les déchets hospitaliers solides sont simplement déversés dans des fosses à brûlage ou même rejetés dans l'environnement sans aucun traitement (Ngaroua et al., 2018). Pour limiter les risques, ils sont parfois traités avec de l'hypochlorure de sodium (eau de javel) dans un but de désinfection. Les déchets liquides, quant à eux, sont souvent évacués dans des rigoles ou déversés dans des fosses d'enfouissement (Ngaroua, 2018).

Dans la ville de Yaoundé, les établissements de soins produisent des déchets en grande quantité et de nature très diverse. Ces déchets à risques représentent 23,21\% des déchets municipaux. Ils sont classés en plusieurs catégories dont les déchets à risque infectieux (DASRI), chimiques toxiques ou radioactifs, pointus ou tranchants, anatomiques et pharmaceutiques. La gestion des $\mathrm{DHs}$ devient une préoccupation majeure aussi bien pour les populations, les collectivités locales que le gouvernement. Cette situation justifie la réalisation de la présente étude dont l'objectif est d'effectuer une analyse de la chaîne de gestion des DHs, partant des systèmes de production jusqu'à leur élimination.

\section{MATERIEL ET METHODES}

\subsection{Matériel}

Dans le but d'atteindre les objectifs fixés, plusieurs outils ont été utilisés :

- Un GPS de marque GARMIN et caractéristiques Etrex 20,64sx, pour les relever des coordonnées géographiques ayant permis de ressortir la carte de localisation de la zone d'étude ;

- Des fiches d'enquêtes permettant de collecter les informations nécessaires portant en la gestion des DHs solides dans les différentes FOSAs de la ville de Yaoundé ;
- Les équipements de protection individuelle (EPI) : gants, chaussures de sécurité, cache-nez, lunettes de laboratoire, blouse et une solution désinfectante dont l'alcool à $90^{\circ} \mathrm{C}$;

- La matrice de Léopold (Léopold et al., 1971) pour la caractérisation des impacts socioenvironnementaux liés à la gestion des $\mathrm{DH}$ s dans les différentes FOSAs de la ville de Yaoundé ;

- Les logiciels de traitement des données et d'analyse des résultats : SPSS, Archi-Card, ArGis et Excel.

\subsection{Démarche méthodologique}

Cette étude a porté sur cinq FOSAs considérées comme représentatives : I'hôpital central de Yaoundé (HCY), I'hôpital Jamot de Yaoundé (HJY), l'hôpital de district de Biyem-Assi (HDB), le centre médical d'Ahala (CMAAhala) et le centre de santé intégré de Mimboman (CSIMimboman). Deux principales raisons expliquent ce choix de la zone d'étude. D'abord, chaque FOSA appartient à une catégorie bien précise parmi les six catégories qui constituent l'ensemble des FOSAs. Ensuite, chaque FOSA appartient à un district de santé parmi les cinq districts de santé que compte la ville de Yaoundé. Ceci dans le but d'établir une bonne traçabilité des DHs dans chaque district de santé. Du fait que dans la ville de Yaoundé il n'existe pas des FOSAs de troisième catégorie, mais aussi du fait que l'accès à l'information dans les FOSAs de première catégorie ne soit possible que sous l'autorisation du Ministre de la santé publique, cette étude a porté exclusivement sur les FOSAs de seconde, quatrième, cinquième et sixième catégorie.

La démarche méthodologique utilisée a consisté en la recherche bibliographique, les investigations sur le terrain puis le traitement et l'analyse des données. L'étape portant sur la recherche bibliographique a été basé en la collecte des informations sur l'origine des DHs solides, la typologie, les différents modes de gestions (Organisation Mondiale de la Santé et al., 1999 ; Ngaroua, 2018) ainsi que les impacts sociaux et environnementaux de la gestion des $\mathrm{DH}$ s dans le monde en général et au Cameroun en particulier (Messi et Djenadek, 2016; Ngaroua, 2018). Les travaux de terrain ont porté essentiellement sur la collecte des données nécessaires à la réalisation effective de l'étude. Cette phase de l'étude comprend d'abord les enquêtes suivies des entretiens personnels auprès de l'ensemble des usagers des FOSAs de notre choix, ensuite des observations et prises de vue, et enfin, la prise des coordonnées géographiques. Pour le traitement et l'analyse des données, les informations collectées lors des investigations sur le terrain ont été traitées par le logiciel SPSS pour certaines et Excel pour d'autres. 


\section{RESULTATS}

\subsection{Etat des lieux préliminaire de la gestion des déchets solides à Yaoundé}

Les observations faites lors des investigations sur le terrain révèlent dans l'ensemble des cinq FOSAs, un espace insalubre inapproprié non seulement pour les malades, mais aussi pour l'ensemble du personnel médical et les populations. Pour ce qui est du niveau de connaissance du personnel médical sur la typologie des DHs solides, l'on note certains manquements au niveau du tri, résultat analogue à celui de Balekouzou (2016) en Inde. Néanmoins, bien que ces derniers n'aient pas $\mathrm{d}^{\prime}$ information sur la typologie générale des $\mathrm{DHs}$, force est de constater que, quelle que soit la catégorie de la FOSA, le personnel médical fait une distinction nette entre les déchets piquants et les déchets tranchants et coupants. En outre, plus de $95 \%$ du personnel des FOSAs de deuxième et quatrième catégorie sont conscients des risques liés à la mauvaise gestion des DHs et plus de $97 \%$ proposent, parmi les meilleures approches de gestion de ces déchets, la séparation à la source. En revanche, près de $50 \%$ du personnel médical des cinquième et sixième catégories des FOSAs n'est pas assez informé des risques liés à la mauvaise gestion des DHs.

\subsection{Gestion des DHS dans les FOSAs de Yaoundé}

\subsubsection{Estimation de la quantité de déchets produite et organisation du système de gestion des DHs dans les FOSAs de la ville de Yaoundé}

L'analyse des données collectées sur le terrain dans diverses catégories de FOSAs de la ville de Yaoundé nous a permis d'évaluer la proportion des différents types de DHs solides qu'on y rencontre en fonction de leur degré de dangerosité et de la catégorie de FOSA (Tableau I). Le Tableau I montre que la grande partie des DHs correspond aux déchets assimilables aux ordures ménagères (DAOMs), les déchets spéciaux étant produits en de très faibles proportions (Figure 1). Sur le plan organisationnel, le système de gestion des DHs solides dans les FOSAs de la ville de Yaoundé est encore à son état fotal pour les FOSAs de deuxième et quatrième catégories et à l'état embryonnaire pour celles des cinquième et sixième catégories.

\subsubsection{Différentes étapes de gestion des DHs dans les FOSAs de la ville de Yaoundé}

Les DHs produits dans les FOSAs doivent toujours suivre un itinéraire approprié et bien identifié de leur point de production à leur élimination finale. Tel que l'ont aussi constaté, Messi et al. (2016), cet itinéraire est composé de plusieurs étapes (Tableau II). Le tri est l'étape de gestion des déchets pratiqués dans toutes les FOSAs de la ville de Yaoundé. En revanche, malgré la mise en place des différents conteneurs pour favoriser le tri, des erreurs sont fréquentes lors du tri à la source, où l'on observe le mélange des DASRI aux DAOMs. Ce qui augmente la quantité des DASRI à traiter, qui sont pourtant déversés dans les bacs à ordures placés en des lieux dits poubelles, sous forme de DAOMs. Le conditionnement, représente l'ensemble des opérations consistant à mettre les $\mathrm{DH}$ s sous une forme convenant à leur transport, leur entreposage ou leur stockage. Un mauvais conditionnement des $\mathrm{DHs}$ est observable dans l'ensemble des FOSAs de la ville de Yaoundé. Quant au stockage, la plupart des services cliniques et centres médicaux sanitaires ne possèdent pas de sites de stockage intermédiaires des DHs. Ces derniers sont généralement abandonnés dans des seaux considérés comme des poubelles et placés à l'extérieur de chaque pavillon de l'hôpital (cas particulier de l'HJY), mais aussi tout au long des couloirs et dans les salles des patients (cas des autres FOSAs de notre échantillon). Dans certains cas (cas du CMAAhala), on peut observer des bacs à ordures au plein cœur de la cours d'hôpital ou dans les salles de soins, servant de poubelles communes (Figure 2(a,b).

Pour ce qui est du stockage central, seule l'HCY dispose de lieu de stockage central des déchets provenant de l'activité de l'ensemble des services spécialisés, malgré son faible volume, au regard de la quantité des déchets reçus.

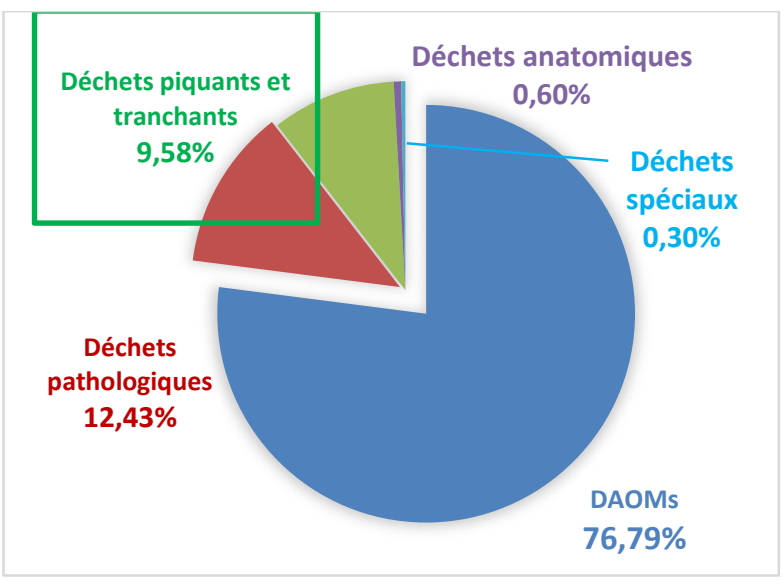

Figure 1. Estimation de la production relative des différents types de déchets dans l'ensemble des FOSAs (formations sanitaires) de Yaoundé (DAOM = Déchets Assimilables aux Ordures Ménagères). 
Tableau I. Estimation de la proportion de déchets produits par catégories dans les FOSAs de Yaoundé.

\begin{tabular}{|l|c|c|c|c|c|}
\hline Types de déchets & HCY & HJY & HDB & CMA-Ahala & CSI-Mimboman \\
\hline Déchets Ménagers et Assimilés & $80 \%$ & $69,24 \%$ & $75,65 \%$ & $80 \%$ & $80 \%$ \\
\hline Pathologiques/infectieux & $19,22 \%$ & $16,81 \%$ & $18,66 \%$ & $3,50 \%$ & $4 \%$ \\
\hline Piquants et tranchants & $0,40 \%$ & $12,54 \%$ & $4,98 \%$ & $15 \%$ & $15 \%$ \\
\hline Anatomiques & $0,26 \%$ & $0 \%$ & $0,71 \%$ & $1,50 \%$ & $1 \%$ \\
\hline Spéciaux & $0,12 \%$ & $1,41 \%$ & $0 \%$ & $0 \%$ & $0 \%$ \\
\hline Total & $100 \%$ & $100 \%$ & $100 \%$ & $100 \%$ & $100 \%$ \\
\hline
\end{tabular}

Tableau 2. Synopsis du parcours des déchets biomédicaux (OMS, 2004)

\begin{tabular}{|c|c|c|c|}
\hline Etape & Lieu & $\begin{array}{l}\text { Parcours des déchets de } \\
\text { soins médicaux }\end{array}$ & Eléments clés \\
\hline 0 & & Minimisation des déchets & $\begin{array}{l}\text { Politiques d'achat, réduction de stocks, recyclage de certains } \\
\text { types de déchets }\end{array}$ \\
\hline 1 & \multirow{2}{*}{$\begin{array}{l}\text { Dans l'unité } \\
\text { médicale }\end{array}$} & & \multirow[t]{2}{*}{$\begin{array}{l}\text { Une des étapes les plus importantes pour réduire les risques à la } \\
\text { quantité des déchets dangereux }\end{array}$} \\
\hline 2 & & Tri à la source & \\
\hline 3 & \multirow{3}{*}{$\begin{array}{c}\text { Dans } \\
\text { I'établissement } \\
\text { sanitaire }\end{array}$} & \multirow{3}{*}{$\begin{array}{l}\text { Collecte et transport } \\
\text { Regroupement sur site } \\
\text { Traitement / stockage } \\
\quad \text { final sur site }\end{array}$} & $\begin{array}{l}\text { Equipements de protection, conteneurs scellés, chariots spéciaux } \\
\text { facile à laver, }\end{array}$ \\
\hline 4 & & & $\begin{array}{l}\text { Salles de stockage fermant à clé. Durée de stockage limitée à } 48 \\
\mathrm{~h}\end{array}$ \\
\hline 5 & & & $\begin{array}{l}\text { Salle de stockage adapté : temps de stockage maximum limite à } \\
48 \text { heures. }\end{array}$ \\
\hline 6 & \multirow{2}{*}{$\begin{array}{l}\text { Dans un site } \\
\text { externe }\end{array}$} & \multirow{2}{*}{$\begin{array}{l}\text { Transport hors site } \\
\text { Traitement / stockage } \\
\text { final sur site }\end{array}$} & $\begin{array}{l}\text { Véhicules et note de colisage appropriée; l'établissement } \\
\text { sanitaire est informé de la destination finale }\end{array}$ \\
\hline 7 & & & ge appropriée pour garantir... \\
\hline
\end{tabular}

\subsection{Impacts socio-environnementaux de la gestion des DHs à Yaoundé}

Les impacts sanitaires et environnementaux des DHs ont été observés par Adjagodo et al. (2016). Ils regroupent les risques traumatiques et infectieux d'une part, les risques chimiques et d'incendie ou d'explosion d'autre part. Néanmoins, d'autres impacts ont été mis en évidence et mentionnés dans la matrice des interrelations des activités des FOSAs avec le milieu (Tableau 3). La matrice de Léopold (Léopold et al., 1971) a permis de corréler les activités associées à la gestion des $\mathrm{DHs}$ d'une part et les composantes de l'environnement d'autre part. Les sous- ensembles de l'environnement considérés dans la matrice de Léopold sont entre autres l'eau, l'air et le sol pour le milieu physique, la faune et la flore pour le milieu biologique et la santé humaine pour le milieu social. Les tableaux III et IV présentent les interrelations et les interactions possibles entre les activités de gestion des DHs solides et les composantes de l'environnement.
Ainsi, l'étude de la matrice de Léopold montre que, la gestion des $\mathrm{DH}$ a des impacts considérables pour l'environnement et essentiellement les milieux aquatiques et atmosphériques. Ces impacts se produisant sur de très longues durées dans l'eau et à un temps court dans l'air. Globalement, le tableau IV montre qu'en fonction du milieu, la durée de l'impact est soit courte, moyenne ou longue. II met également en évidence la probabilité d'occurrence, la nature ainsi que la valeur même (majeure, faible, etc.) de l'impact. 
Tableau 3. Matrice des interactions des activités de l'hôpital avec les composantes du milieu ( $X=$ Interrelation $n=$ négatif $; \boldsymbol{p}=$ positif)

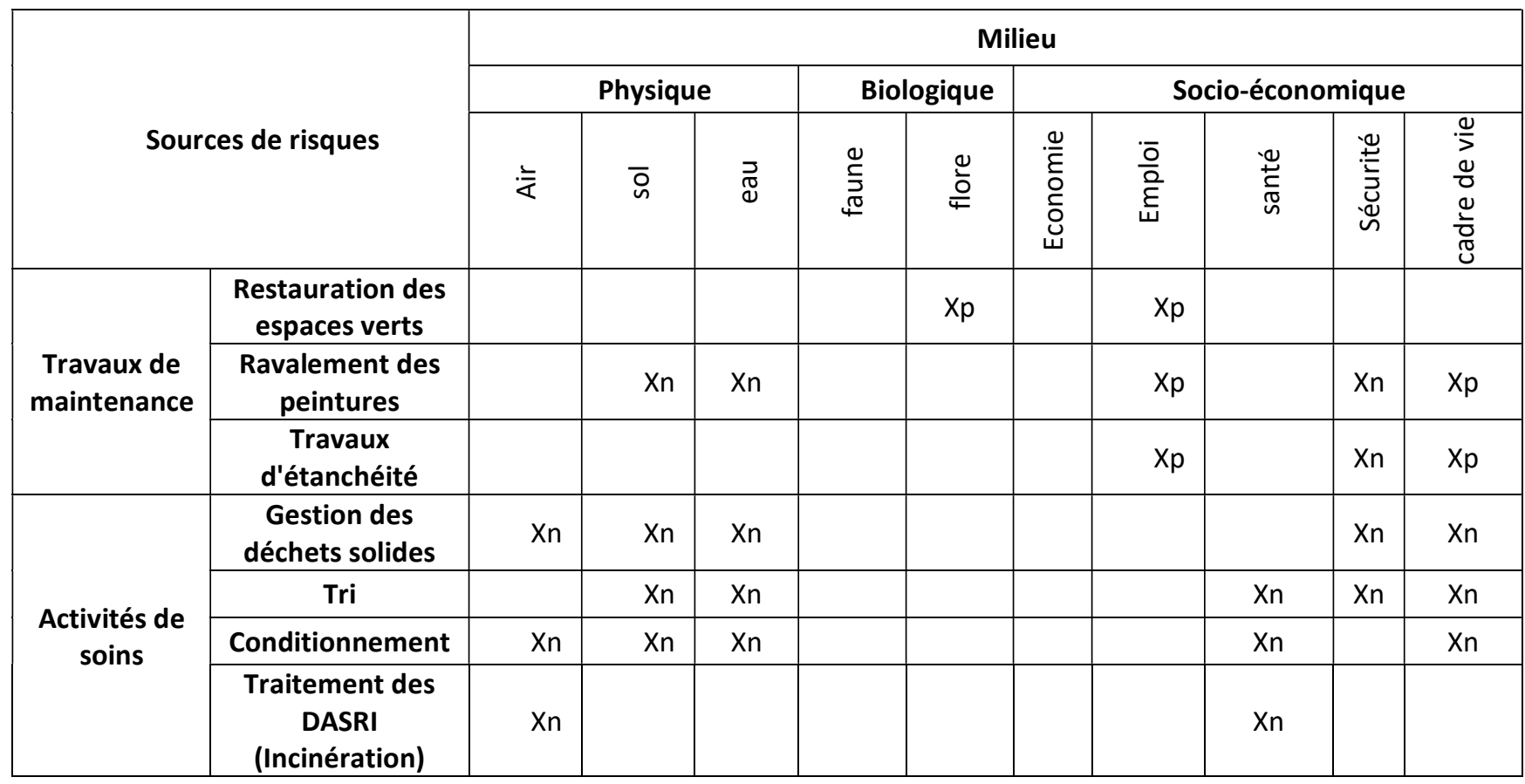

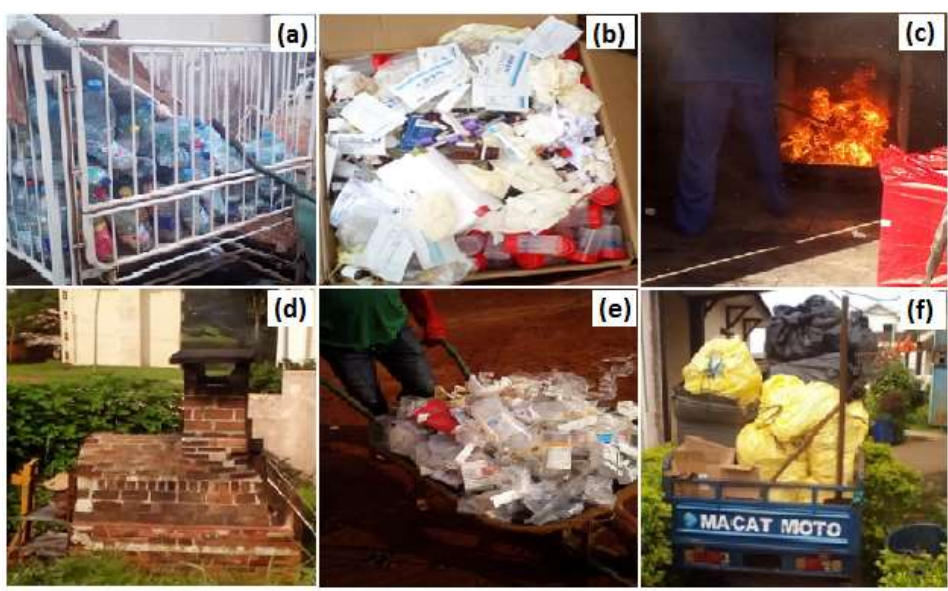

Figure 2. Stockage intermédiaire à (a) : I'HCY ; (b) : I'HJY ; (c) : incinération des DHs au Centre pasteur ; (d) : incinération des DHs à l'HCY ; (e) transport interne à l'aide de la brouette, (f) transport externe à l'aide d'un tricycle 


\section{DISCUSSIONS}

Le manquement observé au niveau du tri est dû à la non-maîtrise des différents types de déchets parmi les DAOMs et les DASRI. Sachant que la gestion des DHs débute par le tri au lieu de production, un système de code de couleur est établi. Malheureusement, dans les FOSAs de la ville de Yaoundé, ce code de couleur n'est pas respecté. Une conséquence directe non seulement du manque de formation et d'information du personnel sur les notions de tri (Sawalem et al., 2009 ; Aseweh et Bouwer, 2008; Daou et al., 2015), mais aussi du manque de protocole et d'affiches portant sur les différentes approches du tri des $\mathrm{DHs}$. Ce qui constitue un danger énorme non seulement pour le personnel médical, mais aussi l'ensemble des usagers des FOSAs et les populations avoisinantes, surtout les enfants qui très souvent fouillent les décharges publiques à la recherche de la ferraille. Dans cet ordre d'idée, Jang (2011) a montré que très souvent, les décharges publiques sont fréquentées par les récupérateurs et beaucoup plus par les enfants pour assurer leur survie tandis que les seringues sont récupérées, nettoyées et réutilisées par les populations (Tamplin et al., 2005).

Les résultats de l'estimation de la quantité des déchets produits dans chaque catégorie de FOSA de la ville de Yaoundé montrent que la majorité des $\mathrm{DH}$ s solides produits (76,98\%) correspondent aux DAOMs.

Seulement 23,02\% correspond aux DASRI. Plusieurs raisons (Tableau II) expliquent le mode de gestion des DHs dans les FOSAs de la ville de Yaoundé.

Ces raisons sont en accord avec celles indiquées par Ndie et al. (2016) dans les structures sanitaires de référence du Nord Cameroun. Toutefois, l'organisation de la gestion des déchets est spécifique à chaque FOSA. Ce résultat n'est d'ailleurs pas surprenant car une évaluation faite par l'OMS dans 22 pays en développement a montré que la proportion d'établissements de soin qui n'appliquent pas les méthodes appropriées d'élimination des déchets va de $18 \%$ à $64 \%$.

Tableau 4. Matrice de caractérisation des impacts

\begin{tabular}{|c|c|c|c|c|c|c|c|c|c|c|c|}
\hline \multirow[b]{2}{*}{$\begin{array}{l}\text { Composante } \\
\text { du milieu }\end{array}$} & \multicolumn{11}{|c|}{ Paramètres de caractérisation } \\
\hline & $\begin{array}{l}\text { Activités et sources } \\
\text { d'impacts }\end{array}$ & Impacts & $\begin{array}{l}\frac{0}{3} \\
\frac{\pi}{20} \\
\frac{\pi}{2}\end{array}$ & 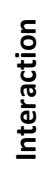 & ڤัٌ & 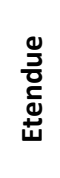 & 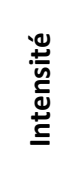 & 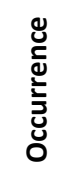 & 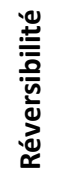 & $\frac{\grave{\partial}}{\frac{\omega}{\pi}}$ & 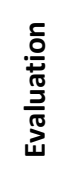 \\
\hline Sol & $\begin{array}{c}\text { Rejet des résidus } \\
\text { d'incinération dans la } \\
\text { nature }\end{array}$ & $\begin{array}{c}\text { Perte du couvert } \\
\text { végétal, pollution } \\
\text { chimique des sols due à } \\
\text { la dégradation des } \mathrm{DHs}\end{array}$ & - & Dir & Cour & Loc & Moy & Cert & Rév & Soc & Moy \\
\hline \multirow[b]{2}{*}{ Air } & $\begin{array}{l}\text { Incinération des } \\
\text { déchets }\end{array}$ & Pollution de l'air & - & Dir & Cour & Loc & Moy & Cert & Rév & Soc & Moy \\
\hline & $\begin{array}{l}\text { Exposition des } \\
\text { poubelles }\end{array}$ & $\begin{array}{c}\text { Emanation des odeurs } \\
\text { nauséabondes et des } \\
\text { gaz toxiques et à effet } \\
\text { de serre tel que le } \\
\text { méthane }\end{array}$ & - & Dir & Cour & Loc & Moy & Cert & Rév & Soc & Moy \\
\hline Eau & $\begin{array}{l}\text { Les eaux de } \\
\text { ruissellement lessivent } \\
\text { des tas d'ordures }\end{array}$ & $\begin{array}{l}\text { Pollution de la nappe } \\
\text { phréatique }\end{array}$ & - & Dir & Long & Loc & For & Prob & Rév & Lég & Maj \\
\hline \multirow{2}{*}{$\begin{array}{l}\text { Environnement } \\
\text { socio- } \\
\text { économique }\end{array}$} & $\begin{array}{l}\text { Rejet anarchique dans } \\
\text { l'enceinte de l'hôpital }\end{array}$ & $\begin{array}{l}\text { Détérioration du cadre } \\
\text { hospitalier et } \\
\text { prolifération des } \\
\text { vecteurs }\end{array}$ & - & Dir & Cour & Loc & For & Cert & Rév & Soc & Maj \\
\hline & $\begin{array}{c}\text { Mauvais } \\
\text { conditionnement }\end{array}$ & $\begin{array}{l}\text { Insalubrité des services } \\
\text { de soins }\end{array}$ & - & Dir & Cour & Loc & Moy & Cert & Rév & Soc & Min \\
\hline
\end{tabular}

- : négatif : Dir : Directe; Indir : Indirecte; Rev : Réversible; Irrév : Irréversible ; + positif ; Loc : Locale ; Leg : Légale ; Soc : Sociale

Cert : Certain ; Moy : Moyen, Maj : Majeur ; Min : Mineur ; Faib : Faible ; Prob : Probable ; Long : Longue ; Cour : Courte ; For : Forte 
L'insalubrité observée dans l'ensemble des FOSAs de la ville de Yaoundé est la conséquence directe du mauvais conditionnement des déchets, de la nonsécurisation des points de stockage primaire des déchets, du disfonctionnement des toilettes et systèmes d'assainissement, de la présence des DHs dans les coins et recoins de l'enceinte des FOSAs considérées. Ces derniers sont à l'origine des émanations désagréables dans l'enceinte des FOSAs de la ville de Yaoundé. L'utilisation des seaux lors du conditionnement, le trop plein des seaux conduisant au déversement des DHs sur le sol, l'absence de couvercles sur les seaux lors du conditionnement pour ne citer que celles-ci, représentent autant de pratiques contraires à la réglementation du domaine. En outre, le compactage interdit lors du conditionnement est aussi une cause du mauvais conditionnement des DHs. La maîtrise de cette étape de gestion des déchets dépend directement des quantités de déchets produites. En revanche, aucune mesure n'est mise en œuvre en vue du contrôle et de la réduction permanente des quantités de DASRI produites. Il en suit donc les difficultés à instaurer et à maintenir les meilleures pratiques du conditionnement des DHs, nécessaires dans l'ensemble des FOSAs de la ville de Yaoundé. La diversification des pratiques de gestion des $\mathrm{DHs}$, au niveau de la production, s'observe par le contenu des déchets produits indépendamment des zones de santé et de la catégorisation des établissements de soins de santé. Ce qui rend difficile l'effectivité d'un système de gestion durable des $\mathrm{DHs}$, pourtant nécessaire dans la capitale politique du Cameroun.

\section{CONCLUSIONS ET RECOMMANDATIONS}

Cette étude établit un état des lieux de la gestion des DHs solides à Yaoundé, intégrant une estimation de la production de déchets par catégorie et par formation sanitaire et une évaluation des sources de production, du matériel de collecte et de regroupement, des sites et des conditions de stockage et d'évacuation. Par une approche transversale descriptive basée sur un choix probabiliste plus qualitatif que quantitatif des FOSAs cibles, l'étude a permis d'avoir une vision complémentaire sur la question des DHs solides, à la fois source de difficultés mais également de dynamisme pour une ville comme Yaoundé en pleine expansion démographique. Globalement, le système de gestion des DHs solides est déficient dans la ville de Yaoundé. L'étude a montré que le tri est une étape de gestion des $\mathrm{DHs}$ pratiquement absente dans le processus de gestion des $\mathrm{DHs}$ dans l'ensemble des FOSAs de la ville de Yaoundé. Cette déficience est aggravée d'une part par leurs rejets dans les décharges municipales d'ordures ménagères et d'autre part par le développement des soins à domicile qui s'accompagnent d'un rejet incontrôlé des $\mathrm{DH}$ s infectieux dans la nature. Un système de gestion durable des DHs, passerait par l'information et la formation non seulement du personnel, mais de l'ensemble des usagers des FOSAs de la ville de Yaoundé, associés à la construction d'un centre de traitement des DHs.

Les problèmes liés à la gestion des $\mathrm{DH}$ s solides dans les FOSAs de la ville de Yaoundé sont très nombreux et divers. Ils sont d'ordre administratif, financier, matériel et humain. En vue d'améliorer le mode de gestion desdits DHs solides dans la ville de Yaoundé, les recommandations suivantes sont formulées :

- La formation du personnel médical aux meilleures pratiques et techniques de gestion des $\mathrm{DHs}$ ainsi que leur information sur les dangers liés à la mauvaise gestion des $\mathrm{DHs}$ solides, la règlementation en vigueur portant sur la gestion des DHs au Cameroun,

- L'information et la sensibilisation des usagers des FOSAs (populations) sur les impacts sociaux et environnementaux de la mauvaise gestion des DHs, ainsi que les meilleures pratiques et comportements à adopter au sein d'une FOSA,

- L'utilisation des bacs de stockage interne mieux adaptés pour la collecte des DHs,

- La construction d'un dispositif de stockage primaire des DHs dans chaque FOSA de la ville de Yaoundé,

- La création d'une unité nationale de pré-collecte des DHs au sein des FOSAs du Cameroun en général et celles de la capitale politique (Yaoundé) en particulier,

- La construction d'un lieu de stockage secondaire dans l'enceinte de l'usine de traitement. Cette dernière étant située en un lieu très éloigné du centre-ville de Yaoundé en vue de protéger les populations de tout risque de contamination. 


\section{REFERENCES BIBLIOGRAPHIQUES}

Adjagodo, A., Tchibozo, M.A.D., Kelome, N.C., Lawani, R. (2016). Flux des polluants liés aux activités anthropiques, risques sur les ressources en eau de surface et la chaîne trophique à travers le monde : synthèse bibliographique. International Journal of Biological and Chemical Sciences, 10(3), 1459-1472, https://doi.org/10.4314/ijbcs.v10i3.43.

Aseweh, A.P., Bouwer, A. (2008). Medical waste management practices in a Southern African hospital. International Journal of Health Care Quality Assurance, 21(4), 356-364.

https://doi.org/10.1108/09526860810880153

Balekouzou, A., Pamatika, C.M., Nambei, S.W., Djeintote, M., Mossoro, D., Ditu, K., Cavin, B., Sume, E.G., Okomo, M.C., Koffi, B., Ping, Y. (2016). Management of biomedical waste in two medical laboratories in Bangui, Central African Republic. PanAfrican Medical Journal, 23, 1-15. https://doi.org 10.11604/pamj.2016.23.237.8786

Béguin, M. (2013). L'histoire des ordures : de la préhistoire à la fin du dix-neuvième siècle. Revue en sciences de l'environnement Vertigo, 13(3),

https://doi.org/10.4000/vertigo.14419

Chartier, Y., Emmanuel, J., Pieper, U., Prüss, A., Rushbrook, P., Stringer, R. (2014). In: Safe Management of Wastes from Health-Care Activities. 2nd ed. Geneva, Switzerland : WHO Press (Edited by Chartier, Y., Emmanuel, J., Pieper, U., Prüss, A., Rushbrook, P., Stringer, R., Townend, W., Wilburn, S., Zghondi, R). 146 p.

Daou, H. M., Karam, R., Khalil, S., Mawla, D. (2015).

Current status of dental waste management in Lebanon.

Environmental Nanotechnology, Monitoring \&

Management, 4, 1-5.

https://doi.org/10.1016/j.enmm.2015.04.002

Datta, P., Mohi, G.K., Chander, J. (2018). Biomedical waste management in India: critical appraisal. Journal of

Laboratory Physicians, 10 (1), 6-14.

https://doi.org10.4103/JLP.JLP_89_17

De Silguy, C. (2009). Histoire des hommes et de leurs ordures du moyen âge à nos jours. Paris, éd Le Cherche Midi, $348 \mathrm{p}$.
Jang, Y.C. (2011). Infectious/Medical/Hospital Waste: general characteristics. In: Encyclopedia of Environmental Health, pp : 227-31. https;//doi.org 10.1016/B978-0-44452272-6.00508-0

Léopold, L.B., Clarke, F.E., Hanshaw, B.B., Balsley, J.R. (1971). A procedure for evaluating environmental impact. Geological Survey Circular 645. Washington: U.S. Geological Survey, 13 p. https://doi.org/10.3133/cir645

Li, C.-S., Jenq, F.-T. (1993). Physical and Chemical composition of hospital waste. Infection Control and Hospital Epidemiology, 14 (3), 145-150. https://doi.org 10.1086/646700.

Messi, P.B.E., Djenadek, A. (2016). Gestion des déchets hospitaliers dans la ville de Yaoundé : cas des districts de santé de la cité verte et de Biyem Assi (Cameroun). International Journal of Humanities and cultural studies, 3 (2), 1153-1176.

Ndie, J., Nongsi, H.B.N. (2016). Etude de la gestion des déchets hospitaliers dans les structures sanitaires de référence de la Région du Nord-Cameroun. European Scientific J., 12 (11), 364-380.

https://doi.org 10.19044/esj.2016.v12n11p364

Nema, S.K., Ganeshprasad, K.S. (2002). Plasma pyrolysis of medical waste. Current Science, 83 (3), 271-278.

Ngaroua, D., Zap, N.N., Dah'Ngwa, D., Yaouba, D., Ngah, J.E. (2018). Knowledge, attitude and practice of health personnel of the regional hospital of Ngaoundere about the management of hospital waste products. Health Sciences and Diseases, 19(3). 111-115.

OMS (Organisation mondiale de la Santé) (1999). Principes directeurs pour l'élimination sans risques des produits pharmaceutiques non utilisés pendant et après les situations d'urgence. Genève NWHO/EDM/PAR/99.2. pp : 1-33.

Sawalem. M, Selic, E., Herbell, J.-D. (2009). Hospital waste management in Libya: A case study. Waste Management, 29 (4), 1370-75.

https://doi.org/10.1016/j.wasman.2008.08.028

Tamplin, S.A., Davidson, D., Powis, B., O'Leary, Z. (2005). Issues and options for the safe destructions and disposal of used injection materials. Waste Management, 25, 655665. https://doi.org/10.1016/i.wasman.2004.07.007 